\title{
Factors Associated With Adequacy of Diagnostic Workup After Abnormal Breast Cancer Screening Results
}

\author{
Mario Schootman, PbD, Jill Myers-Geadelmann, RN, and Laurence Fuortes, MD
}

Background: Women with certain characteristics, such as those residing in rural areas, are less likely screened for breast cancer. To enhance detection of early breast cancer, it is imperative that all women who have abnormal screening results receive appropriate diagnostic procedures. This study reports differences in receipt of diagnostic services following abnormal screening results.

Methods: Screening and diagnostic data were collected as part of a breast and cervical cancer early detection program aimed at reaching women of lower socioeconomic status. Women with completed diagnostic information after having abnormal screening results were included. We based adequacy of diagnostic services on guidelines from the Society for Surgical Oncology, The Commission on Cancer of the American College of Surgeons, and the Centers for Disease Control and Prevention. Several factors were assessed for their association with adequacy of diagnostic follow-up: income, age, race, education, health insurance status, rural-urban residence, reported breast lump, family history of breast cancer, and clinical beast examination or mammogram results.

Results: Overall, 14.1\% of the 351 abnormal findings were considered inadequately followed up based on the algorithm used. Eighty percent involved an abnormal finding on a clinical breast examination regardless of the mammogram results. Rural women, those with abnormal clinical breast examination findings but normal or equivocal findings on mammograms, and those who self-discovered a mass were less likely to receive adequate follow-up than were their counterparts in multivariate analysis. Rural women were less likely to receive a biopsy or fine-needle aspiration, although it was indicated. One facility accounted for most of the inadequate follow-up screenings among urban women.

Conclusions: Women who have specific demographic and clinical characteristics were less likely to have received adequate diagnostic services. Breast cancers could have been missed initially as a result of inappropriate follow-up. Further investigation of the clinical scenarios using chart reviews is warranted. (J Am Board Fam Pract 2000;13:94-100.)

Screening for breast cancer is considered beneficial when conducted at recommended intervals. ${ }^{1} \mathrm{Un}$ fortunately, not all women receive screening to an equal extent. Women who have specific characteristics, such as occupying a lower socioeconomic

Submitted, revised, 21 September 1999.

From the Division of Health Behavior Research (MS), Departments of Internal Medicine and Pediatrics, Washington University School of Medicine, and The Alvin J. Siteman Cancer Center at Barnes-Jewish Hospital (MS), St Louis, Mo; the Bureau of Health Promotion (MS, JM-G), Iowa Department of Public Health. Des Moines; and the Department of Epidemiology (LF), College of Public Health, The University of Iowa, Iowa City, Iowa. Address reprint requests to Mario Schootman, PhD, Division of Health Behavior Research, Departments of Internal Medicine and Pediatrics, Washington University School of Medicine, 4444 Forest Park Parkway, Box 8504, St. Louis, MO 63108 . status, being limited physically, belonging to a minority group, and residing in rural areas, were less likely to be screened for breast cancer. ${ }^{2}$ Reasons for lower screening utilization include fewer primary care physicians in rural areas and access to those physicians, in particular because the clinician's recommendation is the main reason women receive mammograms. ${ }^{3}$

To enhance survival following detection of breast cancer, all women need to receive timely and appropriate diagnostic services based on their screening results. ${ }^{4}$ Delay in establishing a diagnosis and initiating treatment can result in more advanced disease at the time of diagnosis and a worse outcome. Delayed diagnosis also accounts for the most expensive and most common medicolegal claims against physicians. ${ }^{5,6}$ The receipt of timely 
services after abnormal screening results is influenced by such factors as screening results, clinic access, the number of diagnostic events per visit, and access to specialty care ${ }^{7}$ as well as patient characteristics. $^{8}$

Appropriate diagnostic services are essential components of a timely follow-up. Specific diagnostic tests must be completed for the evaluation and management of such breast problems as a palpable mass or a nonpalpable mammographic abnormality. ${ }^{9}$ For example, Osuch et $\mathrm{al}^{5}$ suggest using a triple diagnosis in the management of a solid breast mass. This dramatically increases diagnostic accuracy. If a mass is interpreted as benign by all three methods (follow-up clinical breast examination, mammogram, and fine-needle aspiration), diagnostic accuracy approaches $99 \% .{ }^{10,11}$

Additionally, the use of subsequent diagnostic procedures is imperative if there are abnormal findings on a clinical breast examination and a normal mammogram. A number of breast cancers might be missed because it is not possible to capture the posterior portion of the breast on the film, because a cancer cannot be visualized against a background of dense tissue, or because the radiologist misinterprets the film. ${ }^{11,12}$ Normal mammography findings in the presence of a palpable mass add no information.

A common dilemma facing clinicians is how to approach a patient-discovered mass that is confirmed by physical examination but not visualized on a mammogram. ${ }^{5}$ In the Physician's Insurance Association of America 1995 study, 60\% of women who brought successful claims for failure to diagnose breast cancer had self-discovered masses that failed to impress their physicians on clinical examination; $80 \%$ had normal or equivocal mammogram results. ${ }^{13}$

As with screening utilization, women who share specific demographic characteristics might be less likely to receive follow-up diagnostic services. Hence, our study will attempt to determine which women with questionable breast-screening results are less likely to receive diagnostic services. Specifically we will compare diagnostic services received by rural and urban women. We will also examine diagnostic service utilization by those who come to the clinician's office having found a breast lump and by those for whom findings on the clinical breast examination were abnormal and the mammogram findings were normal.

\section{Methods}

\section{Iowa Breast and Cervical Cancer Early Detection \\ Program}

The Iowa Breast and Cervical Cancer Early Detection Program (BCCEDP) was established to screen eligible women for breast and cervical cancer. Eligibility is based on household income, with women at or below $250 \%$ of the federal poverty guideline eligible to receive services at no cost. Emphasis is placed on screening women aged 50 years and older, although a percentage of women screened are younger than 50 years.

Clinical services were delivered at the local level by more than 300 clinics, mammography facilities, hospitals, and cytology laboratories. Women received usual care from the clinicians. Every woman had a primary care physician who was responsible for her care. No attempt was made to influence delivery of screening and diagnostic work-up.

Data were collected by the Iowa BCCEDP as part of a clinical service delivery monitoring effort. The Iowa BCCEDP consists of 26 local programs spanning 49 counties, one half of all Iowa counties. Each local program is responsible for collecting data about women for which it is administratively responsible.

Two forms were used for data collection, an Intake and Visit Summary form and a Diagnostic Results form. The intake form is completed for each woman regardless of the type of screening service performed. Information contained on this form includes demographics (name, birth date, address, race, ethnicity, highest educational level, household income), history (most recent screening), self-reported breast symptoms, and breast and cervical screening information (type, date and result of clinical service). Initial mammographic findings were reported using the Breast Imaging Reporting and Data System (BI-RADS $\left.{ }^{\mathrm{TM}}\right) \cdot{ }^{14}$ Clinical breast examination findings were reported as normal (not suspicious for cancer) or abnormal (suspicious for cancer).

Diagnostic information is completed when an abnormality is detected at screening. The Diagnostic Results form contains the woman's identifying information and the types, dates, and results of the diagnostic services performed. A final diagnosis and the diagnostic disposition are also included on the form. 
Table 1. Result of Clinical Breast Examination and Mammogram (n - 351) and Adequacy of Follow-Up.

\begin{tabular}{|c|c|c|c|c|}
\hline Algorithm & $\begin{array}{c}\text { Number } \\
\text { (Rural, Urban) }\end{array}$ & CBE Result & Mammogram Result & Diagnostic Procedures Required \\
\hline 1 & $\begin{array}{l}122 \\
(57,65)\end{array}$ & Abnormal, suspect cancer & $\begin{array}{l}\text { Negative } \\
\text { Benign } \\
\text { Probably benign } \\
\text { Assessment incomplete }\end{array}$ & $\begin{array}{l}\text { At least one } \\
\text { Repeat breast examination } \\
\text { Surgical consultation } \\
\text { Sonogram } \\
\text { Biopsy or lumpectomy } \\
\text { Fine-needle or cyst aspiration }\end{array}$ \\
\hline 2 & $\begin{array}{c}36 \\
(26,10)\end{array}$ & Abnormal, suspect cancer & $\begin{array}{l}\text { Suspect abnormality } \\
\text { Highly suggestive of } \\
\text { malignancy }\end{array}$ & $\begin{array}{l}\text { At least one } \\
\text { Biopsy or lumpectomy } \\
\text { Fine-needle or cyst aspiration }\end{array}$ \\
\hline 3 & $\begin{array}{c}83 \\
(65,18)\end{array}$ & $\begin{array}{l}\text { Normal (do not suspect } \\
\text { cancer) }\end{array}$ & Suspect abnormality & $\begin{array}{l}\text { At least one } \\
\text { Repeat breast examination } \\
\text { Surgical consultation } \\
\text { Sonogram } \\
\text { Biopsy or lumpectomy } \\
\text { Fine-needle or cyst aspiration }\end{array}$ \\
\hline 4 & $\begin{array}{l}12 \\
(5,7)\end{array}$ & Any result & $\begin{array}{l}\text { Highly suggestive of } \\
\text { malignancy }\end{array}$ & $\begin{array}{l}\text { At least one } \\
\text { Biopsy or lumpectomy } \\
\text { Fine-needle or cyst aspiration }\end{array}$ \\
\hline 5 & $\begin{array}{c}98 \\
(54,44)\end{array}$ & $\begin{array}{l}\text { Normal (do not suspect } \\
\text { cancer) }\end{array}$ & Assessment incomplete & $\begin{array}{l}\text { At least one } \\
\text { Additional mammographic views } \\
\text { Sonogram }\end{array}$ \\
\hline
\end{tabular}

CBE-clinical breast examination.

Data included in the analysis are those for which a final diagnosis is available during the period from August 1995 through September 1998. Only women with completed follow-up were included in the analysis, and those lost to follow-up or who had refused diagnostic procedures were excluded. Although not all diagnostic services were reimbursed by the Iowa BCCEDP, fine-needle aspiration, additional mammographic views, and breast biopsies were covered services.

A total of 3198 clinical breast examinations and mammograms were performed during the study period, and an abnormality was detected during 351 of the breast examinations or mammograms. Diagnostic procedures were pending for 9 women, 3 women were lost to follow-up, and 5 women refused all diagnostic services. Thus, $95.6 \%$ of women received diagnostic follow-up services.

\section{Definition of Adequacy}

For the current study, we defined adequacy of diagnostic services according to Evaluation of Common Breast Problems: A Primer for Primary Care Providers prepared by the Society for Surgical Oncology, The Commission on Cancer of the American College of Surgeons, and the Centers for Disease Control and Prevention. 'This descripton is a modification of a previously developed algorithm. ${ }^{15}$ Table 1 lists the diagnostic procedures considered to be adequate based on the results of the clinical breast examination and mammogram. Claims data, submitted independently for provided services by the clinics, were used to corroborate the services reported on the submitted Diagnostic Results form.

\section{Statistical Analysis}

Because of the prospective nature of the study, we modeled relations between variables and the dependent variable (adequacy) using relative risks. The proportional hazard approach was used to select factors associated with inadequately performed follow-up services.

Variables that were considered included income category ( $<100 \%$ of poverty vs $101 \%$ to $250 \%$ of federal poverty guidelines), age groups (younger than 50 years vs 50 years and older), race (white vs 
Table 2. Frequency of Inadequate Follow-Up and Associated Factors Using the Proportional Hazard Approach.

\begin{tabular}{|c|c|c|c|}
\hline Demographic Characteristics & Percent & Relative Risk & $\begin{array}{l}\text { Likelihood Ratio Test } \\
\text { ( } P \text { Value) }\end{array}$ \\
\hline Age group & & & $3.92(.0478)$ \\
\hline$<50$ years & 19.0 & $1.75(1.01-3.06)$ & \\
\hline$\geq 50$ years & 10.9 & 1.00 & \\
\hline Race & & & $0.16(.6926)$ \\
\hline White & 14.4 & $1.22(0.44-3.39)$ & \\
\hline Not white & 11.8 & 1.00 & \\
\hline Household income & & & $0.48(.4881)$ \\
\hline$<100 \%$ & 15.3 & $1.22(0.70-2.11)$ & \\
\hline $100 \%-250 \%$ & 12.9 & 1.00 & \\
\hline Education level & & & $0.57(.7527)$ \\
\hline$<$ High school & 13.0 & $1.20(0.56-2.57)$ & \\
\hline High school or GED & 15.6 & $0.95(0.42-2.17)$ & \\
\hline$>$ High school & 12.4 & 1.00 & \\
\hline Geographic location & & & $1.19(.2749)$ \\
\hline Rural & 15.9 & $1.38(0.77-2.48)$ & \\
\hline Urban & 11.6 & 1.00 & \\
\hline Medical insurance & & & $1.01(.3142)$ \\
\hline Yes & 15.4 & $1.40(0.71-2.72)$ & \\
\hline No & 11.0 & 1.00 & \\
\hline Family history of breast cancer & & & $1.50(.2204)$ \\
\hline Yes & 11.0 & $0.69(0.38-1.26)$ & \\
\hline No & 15.8 & 1.00 & \\
\hline Breast symptoms & & & $6.17(.0130)$ \\
\hline Lump & 22.7 & $2.08(1.18-3.64)$ & \\
\hline Other or none & 10.9 & 1.00 & \\
\hline Screening scenario & & & $7.97(.0048)$ \\
\hline Algorithm 1 & 22.1 & $2.23(1.28-3.89)$ & \\
\hline Algorithm 2-5 & 9.9 & 1.00 & \\
\hline
\end{tabular}

GED-general equivalency diploma.

other), education (less than high school, high school, or general equivalency diploma, more than high school), having health insurance (yes or no), resident of a metropolitan statistical area (MSA) or not, reported breast lump (yes or no), family history of breast cancer (yes or no), and clinical breast examination or mammogram results (algorithm 1 vs algorithm 2 to 5). Iowa has 10 metropolitan statistical areas. These counties were considered urban for the study, whereas the other 89 were considered rural.

A best regression model was created using two approaches. We used the likelihood ratio test comparing a model with and without a particular variable. We considered $P \leq .05$ to be statistically significant. A variable could also be included based on important changes in the regression coefficients of a model with and without this variable.

\section{Results}

Overall, $14.1 \%$ of the 351 abnormal screenings were considered inadequately followed up based on the algorithms listed in Table 1. Table 2 lists the percentage of inadequate follow-up services for various demographic and clinical variables. Variables that are potentially associated with inadequate follow-up include age-group, having felt a lump, and having screening results according to algorithm 1. In this univariate analysis, household income, highest level of education attained, race, family history of breast cancer, having health insurance, and the residence of the woman did not affect subsequent adequacy of follow-up services.

Using the proportional hazard approach, the model included three variables. Rural women were less likely than urban women to receive adequate diagnostic services $(\mathrm{RR}=0.56 ; 95 \% \mathrm{CI}, 0.30-$ 


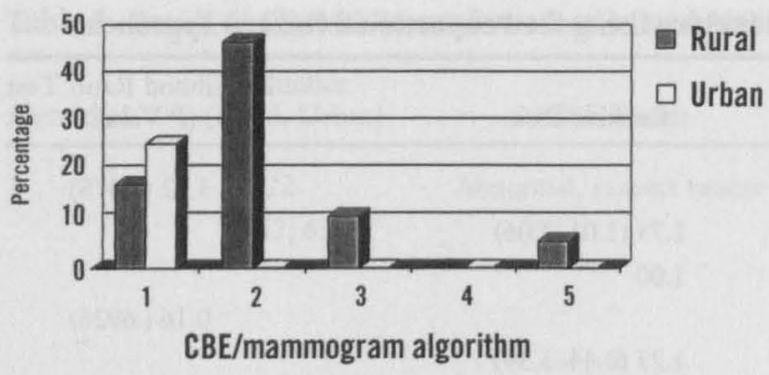

Figure 1. Percentage of inadequately followed up abnormal breast cancer screening results, by clinical breast examination and mammogram results.

1.03). Additionally, women who had screening results listed in algorithm 1 were 0.47 times as likely to receive adequate follow-up services compared with those with other clinical breast examination and mammogram results (95\% CI, $0.26-0.88$ ). The interaction between geographic location and type of clinical breast examination or mammogram result could not be assessed because all urban women with inadequate follow-up services had an abnormal clinical breast examination and normal mammogram findings (Figure 1).

Although having felt a lump on breast self-examination did not statistically predict adequacy of follow-up after inclusion of the clinical breast examination or mammogram results and the residence of the woman screened, the regression coefficients of the model containing both variables changed to an important degree. Women who felt a lump were 0.57 times ( $95 \% \mathrm{CI}, 0.30-1.06$ ) as likely to have received adequate follow-up services than those who did not have any symptoms.

The need to assure appropriate diagnostic services for a woman who self-discovers a mass, which is confirmed by the clinician, but who has subsequent normal findings on a mammogram is selfevident. $^{9}$ Women with these results were 0.27 times as likely to have adequate follow-up services as those who did not feel a mass on examination and had other clinical breast examination and mammogram results.

\section{Types of Inadequate Diagnostic Workup}

Of all screenings among women who did not receive appropriate follow-up according to the algorithm used ( $\mathrm{n}=50$ ), $80.0 \%$ involved an abnormal clinical breast examination finding regardless of the result of the mammogram (algorithm 1 or 2). Differences in inadequate follow-up were found be- tween rural and urban women. Among rural women with abnormal findings on a clinical breast examination and mammograms, $48.0 \%$ did not receive a biopsy or lumpectomy or a fine-needle or cyst aspiration while indicated by the algorithm. In some cases, rural women with other clinical breast examination and mammogram results did not receive appropriate diagnostic follow-up, such as for algorithm 1 and 3 .

Among urban women all inadequate follow-up services were for those who had abnormal findings on a clinical breast examination and normal findings (or assessment incomplete) on a mammogram (algorithm 1). In contrast, rural women had a variety of screenings for which they received inadequate follow-up services.

\section{Discussion}

The aim of our study was to assess factors associated with women of lower socioeconomic status receiving adequate diagnostic services after abnormal results on breast cancer screening. Ninety-six percent of women with abnormal findings on breast screening received diagnostic procedures, which indicates that even women of lower socioeconomic status can receive these services when indicated. This percentage of women receiving diagnostic workup is much higher than that reported by a study using BCCEDP data in California ${ }^{16}$ and is necessary to maximize screening benefits.

Rural women, those with abnormal clinical breast examination results and normal mammogram results, and those who self-discovered a breast mass were less likely than their counterparts to receive an adequate diagnostic workup based on guidelines prepared by the Society for Surgical Oncology, The Commission on Cancer of the American College of Surgeons, and the Centers for Disease Control and Prevention. ${ }^{9}$ None of the other variables were associated with less-than-adequate follow-up services. Even women without health insurance, minority women, and those living in poverty received diagnostic services similar to those received by their counterparts. Because women with these characteristics are typically less likely to be screened for breast cancer, our study shows that it is possible to assure adequate follow-up diagnostic services for these women.

Rural women were less likely to receive appropriate diagnostic services in our study. The main 
reason for inadequate follow-up among rural women is underuse of biopsy and fine-needle or cyst aspiration among those with both abnormal findings on clinical breast examinations and mammograms (algorithm 2). A proportion of these women might have had their condition initially misdiagnosed as not being breast cancer and later have more advanced disease.

In contrast with the distribution of the percentage of rural women with inadequate follow-up (Figure 1), all urban women with inadequate diagnostic services had abnormal clinical breast examination findings and normal findings on a mammogram (or assessment incomplete) as described in algorithm 1. On closer examination, one clinic site accounted for almost three out of every four screenings.

Based on the rural-urban differences and the percentage of inadequate follow-up services for each of the five algorithms, it can be concluded that assurance of appropriate follow-up is warranted when there are abnormal findings on clinical breast examination and normal findings on a mammogram. A significant percentage of breast cancers will be missed when not performing adequate workup of a breast mass. ${ }^{9}$ Increasing emphasis has recently been placed on this clinical scenario, because not all clinicians are familiar with the appropriate guidelines to maximize breast cancer detection and evaluation.

In our study, a patient with a self-discovered mass was less likely to receive adequate diagnostic services independent of the other two variables included in the regression model. Concern is warranted regarding these results, which need further investigation. Cultural and psychosocial factors might play a role. ${ }^{17}$ It is not possible to compare our findings with those of other studies because there is too little information. Nevertheless, incorporating risk management strategies, such as taking the complaint seriously, further physical examination, and careful documentation, is appropriate. ${ }^{5}$

This study has its strengths and limitations. One strength is that the current study included several clinics rather than selected clinics or hospitals, which allowed for a more population-based approach. As a result, we were able to monitor service delivery from a system viewpoint. Second, this study included only women of lower socioeconomic status, a population that has not been exten- sively studied in relation to the diagnostic workup of their abnormal breast screening results.

One limitation of the study is the use of the algorithm. Although several agencies and organizations were involved in the development of the guidelines, it is not possible to describe every clinical situation. It is expected, however, that the overall results did not suffer from this limitation. Moreover, from a system point of view, the results provide valuable insight into where efforts need to be focused.

A second limitation is that the results were based on relatively few cases. Rural-urban differences were close to being statistically significant, however, and we found a lack of appropriate workup among those with abnormal clinical breast examination findings and normal findings on mammograms. If more cases had been included, the results would have been even more pronounced.

In conclusion, the results show important differences in screening results between rural and urban populations. These analyses are only the first step toward assuring that all women receive appropriate diagnostic services. We have planned to investigate further the clinical scenarios by using chart reviews.

\section{References}

1. US Preventive Services Task Force. The guide to clinical preventive services, 2nd ed. Alexandria, VA: International Medical Publishing, 1996.

2. Schootman M, Fuortes L. Breast and cervical cancer: The relationship of activity limitations and rurality with screening, incidence, and mortality. Cancer 1999;86:1087-94.

3. Centers for Disease Control and Prevention. Primary care provider adherence to screening guidelines for breast and cervical cancer. A literature review. Washington, DC: Department of Health and Human Services, 1991.

4. Afzelius P, Zedeler K, Sommer H, Mouridsen HT, Blichert-Toft M. Patient's and doctor's delay in primary breast cancer. Prognostic implications. Acta Oncol 1994;33:345-51:

5. Osuch JR, Bonham VL, Morris LL. Primary care guide to managing a breast mass: a legal perspective on risk management. Medscape Womens Health 1998;3:3.

6. Steykal R. Minimizing the risk of delayed diagnosis of breast cancer. Medscape Womens Health 1996; $1: 7$.

7. Wall P, Moore C, El-Tamer M, Reilly JJ. Diagnostic delay in breast disease: a system analysis of a public urban hospital. Arch Surg 1998;133:662-6.

8. Burgess CC, Ramirez AJ, Richards MA, Love SB. 
Who and what influences delayed presentation in breast cancer? Br J Cancer 1998;77:1343-8.

9. Cady B, Steele GD, Morrow M, Gardner B, Winchester DP. Evaluation of common breast problems: A primer for primary care providers. Washington, DC: US Department of Health and Human Services, 1995.

10. Layfield LJ, Glosgow BJ, Cramer H. Fine-needle aspiration in the management of breast masses. Pathol Annu 1989;24(Pt 2):23-62.

11. Donegan WL. Evaluation of a palpable breast mass. N Engl J Med 1992;327:937-42.

12. Svane G, Potchen EJ, Sierra A, Azavedo E. Screening mammography. Breast cancer diagnosis in asymptomatic women. St. Louis: Mosby, 1993.

13. Physician's Insurance Association of America. Breast cancer study. Lawrenceville, NJ: PIAA 1995.
14. Breast Imaging Reporting and Data System (BIRADS $^{\mathrm{TM}}$ ), ed 2. Reston, VA: American College of Radiology, 1995.

15. Smith BL, et al. In: Diercks DB, Cady B: Lawsuits for failure to diagnose breast cancer. Tumor biology in causation and risk management strategies. Surg Oncol Clin N Am 1994;3:125-39.

16. Arnsberger Weber P, Fox $P$, Zhang X, Pond M. An examination of differential follow-up rates in breast cancer screening. J Community Health 1996;21: 123-32.

17. Lannin DR, Matthews HF, Mitchell J, Swanson MS, Swanson FH, Edwards MS. Influence of socioeconomic and cultural factors on racial differences in late-stage presentation of breast cancer. JAMA 1998; 279:1801-7. 